\title{
RAMADAN FASTING EFFECTS ON METABOLIC PARAMETERS IN ELDERLY PERSONS WITH CARDIOVASCULAR RISK FACTORS
}

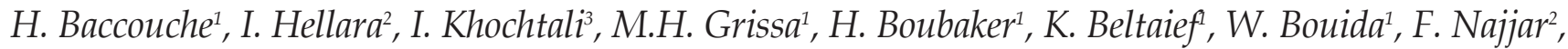 \\ R. Boukef', M. Hassine ${ }^{4}$ S. Nouira ${ }^{1}$ and the Ramadan Research Group
}

\begin{abstract}
Objective: Our study aims was to evaluate the effect of Ramadan fasting on routine biochemical parameters in elderly subjects with cardiovascular risk factors. Design: Cohort study. Setting: Subjects were prospectively recruited and screened at nine primary care clinics, three outpatients specialized clinics and an emergency departement. Participants: subjects aged $\geq 65$ years $(\mathrm{n}=87)$ recruted in Ramadan month in 2010, 2011 and 2012. Measurements: Dietary intake using a 24 hour recall, biochemical tests including complete lipid profile (total cholesterol, triglycerides, high-density lipoprotein cholesterol (HDL-C) and low-density lipoprotein cholesterol (LDL-C), renal function tests, serum uric acid, serum electrolytes (sodium, potassium, chloride and protein), glycaemia, and glycated hemoglobin (HbA1C). All data related to dietary intake and biochemical tests were performed one month before Ramadan, during the last week of Ramadan and one month after Ramadan. Comparison between the three time groups were made using standard statistical tests. Results: We showed a significant decrease of creatinine clearance and an increase of serum triglycerides and blood glucose during Ramadan. After Ramadan, we observed a significant increase in HDL-C was and a significant decrease in serum triglycerides and HbA1c. No correlation was found between glycaemia and total energy intake, neither between LDL-C/HDL-C ratio and total energy intake. Conclusion: In elderly subjects with cardiovascular risk factors, Ramadan seems to induce dual effects. During the fasting period, there is a potential risk of renal function decrease and an increase of glycaemia. In contrast, after Ramadan, our findings support the potential beneficial effect of fasting on lipid regulation and glycemic control.
\end{abstract}

Key words: Ramadan fasting, ageing, nutrition, chemical biology.

\section{Introduction}

Ramadan fasting is one of the five pillars of Islam. It's an intermittent fasting that extends every day from dawn till sunset during one month per lunar year (1). It's different from the physiological fasting by its longer duration and its situation in the circadian cycle. It corresponds to the period where expenditure and energy requirements are the strongest and where the body is «refueled». This holy fasting remains largely followed by Muslims and it has a significant social and economic implications and therefore a new rhythm of life that mainly affects eating habits, sleep and work schedules. These changes have no doubt many biological and physiological consequences on the human body

1. Emergency Department and Laboratory Research (LR12SP18), Fattouma Bourguiba University Hospital and University of Monastir, 5019, Monastir, Tunisia; 2. Biochemistry Laboratory, Fattouma Bourguiba University Hospital, 5019, Monastir, Tunisia; 3. Endocrinology Department, Fattouma Bourguiba University Hospital, 5019, Monastir, Tunisia; 4. Hematology Laboratory, Fattouma Bourguiba University Hospital, 5019, Monastir, Tunisia.

Corresponding Author: Pr. Semir Nouira, Emergency Department and Laboratory Research (LR12SP18), Fattouma Bourguiba University Hospital, University of Monastir, 5019, Monastir, Tunisia. +216 73106 046. e-mail : semir.nouira@rns.tn
$(2,3)$. The elderly person with his specific physiology and usual polymedication is more sensible than young subjects to the extreme variations in nutriments intakes (fasting or excessive food intake) (4). Although the medical benefits of Ramadan fasting has been suggested in many disorders particularly diabetes, hypertension, congestive heart failure, and psychosomatic diseases (5), few data are available regarding its effects on biochemical parameters in the elderly (6-9), in particular, in presence of cardiovascular risk factors. The aim of our work is to study the effect of Ramadan fasting on some biochemical parameters in 87 aged subjects ( $\geq 65$ years) with cardiovascular risk factors.

\section{Materials and Method}

\section{Subjects}

This was a prospective study conducted during three consecutive Ramadan months that occurred during summer seasons of 2010, 2011 and 2012. We have included 87 patients ( 42 men and 45 women) with a mean 
age $71.6 \pm 5.5$ years. They were recruited from different sites including nine primary care clinics in Monastir area, three outpatient specialized clinics (cardiology, endocrinology, internal medicine) and the emergency department of Fattouma Bourguiba University hospital Monastir (Tunisia). Primary care physicians from the clinical site primary care practice who participate in the study were trained during the previous 6 months to be familiar with the study eligibility criteria, objectives, and what they would be asked to do as a study recruiters. At the time of the clinic enrollment, participants are asked to provide written for the study interventions and all study follow-up assessments.

The study received approval from the Institutional Review Board of our institution. Patients were included in the study if their aged is over 65 years, they have significant cardiovascular risk ( $>20 \%$ Framingham score), or 2 cardiovascular risk, and they plan to fast during Ramadan month. Patients were excluded if they have recent (within the last three months) cardiovascular acute events or an acute comorbidity decompensation.

\section{Methods}

All recruited patients were directed to the principal investigation clinical center of Fattouma Bourguiba University hospital specifically dedicated to the study. Three clinical visits were planned for each included patient: The first visit one month before Ramadan month (baseline); the second visit during the last week of Ramadan, and the third visit one month after the end of Ramadan. Between each visit, the patient received telephonic reminders for their upcoming appointments.

The following clinical data were recorded: demographic characteristics, previous medical history, Framingham score, ongoing treatments, and physical exam data. During each visit, all patients underwent biochemical tests including complete lipid profile (total cholesterol, triglycerides, high-density lipoprotein cholesterol (HDL-C) and low-density lipoprotein cholesterol (LDL-C), renal function tests, serum uric acid, serum electrolytes (sodium, potassium, chloride and protein), glycaemia, and glycated hemoglobin (HbA1C). All the samples were performed on fasting between 8.00 and 10.00 a.m.

After centrifugation, the plasma was frozen and then analyzed on the same day in the Biochemistry department using a Cobas 6000TM analyzer (Roche Diagnostics). Lipid profile was measured by enzymatic colorimetric methods. The LDL-C was calculated by the Friedwald formula. Creatinine was analyzed with Jaffe method and creatinine clearance was calculated by the MDRD simplified formula. Glycaemia and urea was measured by enzymatic on $\mathrm{UV}, \mathrm{HbA} 1 \mathrm{C}$ was analyzed by high performance liquid chromatography. During the three planned visits, we surveyed the patients with a questionnaire elaborated in the Department of clinical dietetics and nutrition. Caloric intake was measured using 3-day diet diaries. Intake assessment was made by the method of current listing of products and beverages consumed in the successive three days. Portion size was estimated by using the "Album of photographs of products and dishes". Using the table of the nutritional value of food products and dishes, we calculated the energy value, content of essential nutriments.

\section{Statistical analysis}

The distribution of the parameters was determined with Kolmogorov Smirnov and Shapiro-Wilk normality test. In normally distributed groups, results were presented as mean \pm standard deviation. To compare mean values of different parameters, we used pairedsamples T-test. For non-normally distributed variables, medians were used and compared by non-parametric Wilcoxon test.

Correlations between energy intake and glycaemia and LDL-C/HDL-C ratio were performed using Pearson or Spearman tests. The data were analyzed by SPSS 18.0. A $p$ $<0.05$ was considered as the cut-off value for significance.

\section{Results}

\section{Patient's characteristics}

87 patients were included in this study, aged $71.6 \pm 5.5$ years old. $57.5 \%$ of them had 2 cardiovascular risks, $25.3 \%$ had 3 cardiovascular risks and $17.2 \%$ had more than 4 cardiovascular risks. The most frequent cardiovascular risk was hypertension ( $86 \%$ ) then dyslipidemia (57\%) and almost one third (30\%) had a previous history of coronary artery disease. Most of our patients were taking aspirin $(74.7 \%)$ or of angiotensin converting enzyme inhibitors $(62.1 \%)$ while the half had oral antidiabetic treatment $(50.6 \%)$.

Demographic and clinical characteristics of the patients are reported in Table 1.

\section{Changes in biochemical parameters}

During Ramadan serum creatinine, glycaemia and serum sodium increased significantly compared to baseline. After Ramadan these parameters showed a significant decrease compared to Ramadan values. Creatinine clearance significantly decreased during Ramadan and increased significantly after Ramadan. For diabetics, glycated hemoglobin did not change significantly during ramadan compared to baseline, but decreased significantly after Ramadan fasting.

With regard to changes in lipid profile, there was no significant change in serum total cholesterol and LDL-C lipids during Ramadan, however, HDL-C levels decreased and serum triglycerides increased significantly. 
After Ramadan, HDL-C levels increased and serum triglycerides decreased significantly.

Table 1

Patients's demographic and clinical characteristics

\begin{tabular}{lc}
\hline Age years, mean \pm SD & $71.6 \pm 5.5$ \\
Gender female $\mathrm{n}(\%)$ & $45(51.7)$ \\
Comorbidity n $(\%)$ & \\
Hypertension & $75(86.2)$ \\
Diabetes & $45(51.7)$ \\
Dyslipidemia & $50(57.5)$ \\
Smoking & $23(26.4)$ \\
Heredity coronary & $26(30.0)$ \\
Cardiovascular risk number n $(\%)$ & \\
2 & $50(57.5)$ \\
3 & $22(25.3)$ \\
$\quad \geq 4$ & $15(17.2)$ \\
Ongoing treatment n $(\%)$ & \\
$\quad$ Aspirin & $65(74.7)$ \\
$\quad$ Angiotensin Converting Enzyme inhibitors & $54(62.1)$ \\
Oral antidiabetics & $44(50.6)$ \\
Statins & $42(48.3)$ \\
$\quad$ Beta blockers & $35(40.2)$ \\
$\quad$ Diuretics & $34(39.1)$ \\
Calcium chanel blockers & $21(24.1)$ \\
Clopidogrel & $14(16.1)$ \\
\hline
\end{tabular}

During and after Ramadan serum albumin levels decreased significantly compared to baseline.

Changes in biochemical parameters are reported in Table 2.

\section{Changes in nutritional intake}

The total energy intake decreased during Ramadan and increased after Ramadan; all these changes were significant. Overall changes of protein, fat and carbohydrate intake percentages were not significant. Neither glycaemia nor the LDL-C/HDL-C ratio was correlated to the total energy intake (Figure 1 and 2).

Changes in nutritional intake are reported in Table 3.

\section{Figure 1}

Correlation between glycaemia and total energy intake. There is no correlation between the glycaemia and the total energy intake

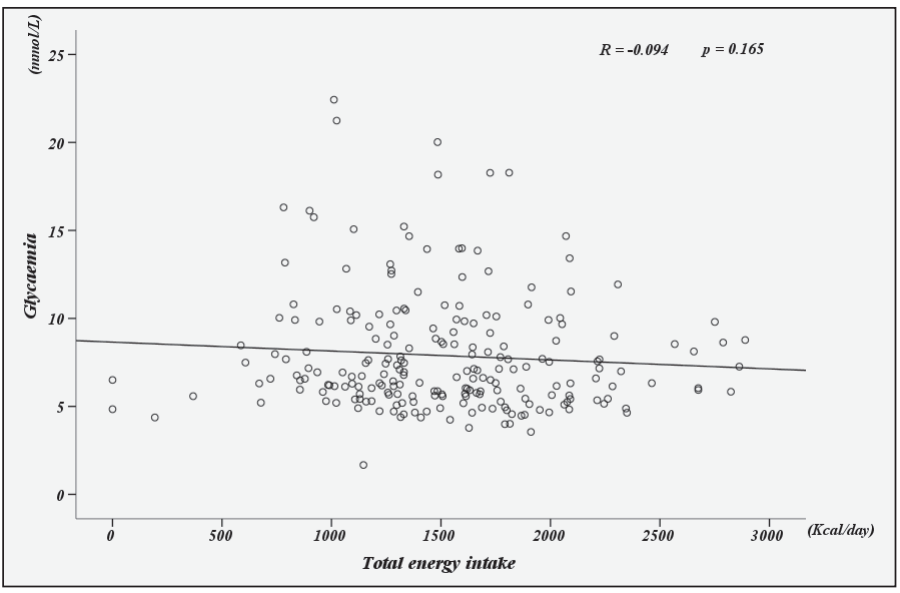

\section{Discussion}

Changes in nutritional habits are known to cause various changes in metabolism. Intermittent daytime fasting during Ramadan which lasts for 1 month causes very important changes in the nutritional status of the fasting people (1). The present study investigates, for the first time, the effect of Ramadan fasting on biochemical parameters in elderly person with significant cardiovascular risk factors. The major finding of our study was the significant increase of creatinine and urea during Ramadan and their significant decrease

Table 2

Changes in serum biochemical parameters before (baseline), during and after Ramadan§

\begin{tabular}{lrrr}
\hline & Baseline & During Ramadan & After Ramadan \\
\hline & & & \\
Creatinine $(\mu \mathrm{mol} / \mathrm{L})$ & $103[69-256]$ & $108^{*}+[74-259]$ & $103[72-273]$ \\
Creatinine clearance $(\mathrm{ml} / \mathrm{min})$ & $52(12)$ & $49^{*}+(11)$ & $7.4^{*}+ \pm 3$ \\
Blood urea nitrogen $(\mathrm{mmol} / \mathrm{L})$ & $6.5 \pm 2$ & $4.62 \pm 1.03$ & $6.3 \pm 2$ \\
Total cholesterol $(\mathrm{mmol} / \mathrm{L})$ & $4.59 \pm 1.07$ & $4.69 \pm 1.09$ & $1.24[0.44-5.37]$ \\
Triglycerides $(\mathrm{mmol} / \mathrm{L})$ & $1.33[0.64-4.51]$ & $2.63 \pm 0.89$ & $2.68 \pm 0.95$ \\
LDL-C $(\mathrm{mg} / \mathrm{L})$ & $2.63 \pm 0.91$ & $1.23+ \pm 0.36$ & $1.34^{* *} \pm 0.41$ \\
HDL-C $(\mathrm{mmol} / \mathrm{L})$ & $1.27 \pm 0.35$ & $7.17^{*}+[1.67-22.43]$ & $6.62[3.55-18.28]$ \\
Glycaemia $(\mathrm{mmol} / \mathrm{L})$ & & $7.05^{* *}[5.2-12.2]$ \\
HbA1c\# $(\%)$ & $7.5[4.01-20.02]$ & $149^{*}+[141-163]$ & $151^{* *}[139-161]$ \\
Sodium $(\mathrm{mmol} / \mathrm{L})$ & $150[141-168]$ & $4.5+ \pm 0.5$ & $4.7 \pm 0.4$ \\
Potassium $(\mathrm{mmol} / \mathrm{L})$ & $4.7 \pm 0.7$ & $106^{*}+ \pm 3$ & $108 \pm 4$ \\
Chlorides $(\mathrm{mmol} / \mathrm{L})$ & $108 \pm 4$ & $69.4^{*}+[57.8-85.1]$ & $68.1^{* *}[57.9-86.9]$ \\
Albumin $(\mathrm{g} / \mathrm{L})$ & $71.1[58.9-89.3]$ & & \\
\hline
\end{tabular}

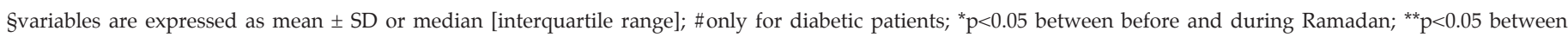
before and after Ramadan; $t \mathrm{p}<0.05$ between during and after Ramadan. 
Table 3

Changes in body mass index, waist size and dietary intake before (baseline), during and after Ramadan§

\begin{tabular}{lrrr}
\hline & Baseline & During Ramadan & After Ramadan \\
\hline & & & \\
Body Mass Index & $30.2 \pm 5.3$ & $29.9 \pm 5.1$ & $30.1 \pm 4.9$ \\
Waist size & $102 \pm 9$ & $100^{*} \pm 7$ & $101^{* *} \pm 8$ \\
Energy intake (Kcal/day) & $1625 \pm 521$ & $1232^{*}+ \pm 411$ & $1714 \pm 438$ \\
Protein $(\%)$ & $17 \pm 4$ & $15 \pm 4$ & $16 \pm 3$ \\
Fats $(\%)$ & $26 \pm 7$ & $25 \pm 10$ & $28 \pm 8$ \\
Carbohydrate $(\%)$ & $57 \pm 8$ & $59 \pm 11$ & $55 \pm 8$ \\
\hline
\end{tabular}

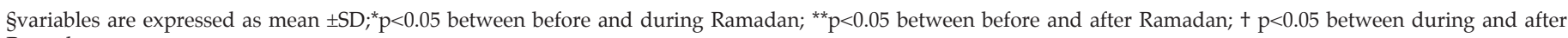
Ramadan.

after the end of the month. Ramadan had no significant effect on the lipid profile except for the triglycerides which increased during Ramadan and decreased after fasting. Simultaneously, HDL-C decreased after Ramadan significantly. Glycaemia increased during Ramadan then returned to baseline values after Ramadan. Glycated hemoglobin did not change during Ramadan but decreased significantly after Ramadan fasting compared with its value before Ramadan. During Ramadan serum sodium increased significantly but serum potassium did not change significantly. We have to notice that the high values of sodium were due to the defrosting effect (10). The dietary intake showed that total energy intake decreased during Ramadan and increased after, but that without a change in the percentage of the different nutrients.

\section{Figure 2}

Correlation between LDL/HDL ratio and total energy intake. There is no correlation between the LDL/HDL ratio and the total energy intake

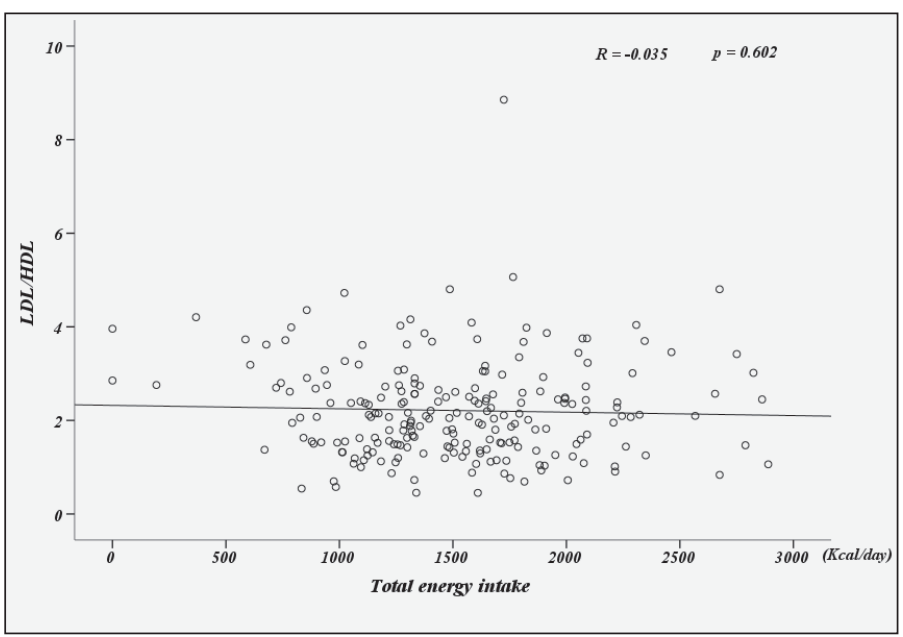

The majority of previous studies investigating metabolic changes during Ramadan had included young and healthy subjects. This population is unlikely to be suitable to detect the potential benefits or harmful effects of prolonged fasting. Only one previous study (4) has been specifically addressed to the elderly person and Ramadan. This cross-sectional Tunisian study was limited to a dietary survey and a collection of anthropometric parameters. It showed that nutritional intake in this population was unbalanced quantitatively and qualitatively during the month of Ramadan. Similar findings were observed by Sebbani et al (6) and others.

Several studies have determined the effects of Ramadan fasting on metabolic parameters with mixed results. El Wakil et al (11) found in 2007 that the Ramadan fast can have a detrimental effect on the renal tubules in patients with chronic renal failure in predialysis stage which may explain the significant decrease in glomerular filtration rate during Ramadan in our patients. Similarly, in 2008, Maughan et al (12) showed an increase in serum creatinine in 78 Tunisian football players (aged 16-19 years old) significantly especially in the 4th week of Ramadan fasting and after 3 week. Trabelsi et al (13) also found a decrease in renal function in bodybuilding coaches during Ramadan and they explained their findings by dehydration. We believe that hypovolemia is the main explanation in our study with regard to the significant increase in serum sodium in our patients resulting from a decrease in water intake and the hot temperature that characterizes the summer in Tunisia. However, the magnitude of the effects on serum creatinine level is modest and not clinically relevant and probably not sufficient to recommend patients to avoid fasting even for those with chronic renal failure or renal transplantation (14).

With regard to the influence of Ramadan fasting on serum lipid, it is important to note that there was a great disparity in the available data ranging from a protective effect and deleterious ones. Recently, a metaanalysis performed by Kul et al (15) including 30 studies, found that the LDL-C decrease after Ramadan while total cholesterol and triglycerides did not change while HDL-C increased. Our results were concordant with these findings regarding HDL-C variations but were different with regard to LDL-C and triglycerides changes. The study of Nematy et al (8) showed mainly a decrease in triglycerides, total cholesterol and LDL-C against a significant increase in HDL-C after Ramadan which suggest Ramadan fasting can be considered as a non- 
pharmacological method to prevent lipid disorders which remained up to one month after the end of Ramadan. The existing controversies in this issue are likely the result of differences in nutrition behavior and life habits that characterizes each population included in the studies conducted during Ramadan. In addition, most of these studies included a reduced sample size population and not specifically designed for elderly. The most important question is whether the metabolic positive findings associated with Ramadan fasting would have any clinical relevance. Some studies have demonstrated a beneficial effect of Ramadan fasting on acute cardiovascular and anthropometric parameters. In a retrospective study conducted by Temizhan et al (16) including 1655 patients, it was found that there was a net decrease in acute coronary artery disease events during Ramadan. In a recent review, Salim et al (17) showed in patients with heart disease that Ramadan fasting was well tolerated and not associated with an increase in decompensation rate. They concluded that these patients can fast without risk except those with diabetes should be carefully monitoring during Ramadan. In fact, several studies have noted an increase in blood glucose during Ramadan (18-21) and a significant decrease after Ramadan (15, 20, 22-24) which is consistent with our results.

The discrepancies in the results among available studies assessing metabolic effects of Ramadan are due to several factors. These factors include differences in protocols, differences in the eating and nutritional customs and habits, difference in climates depending on the location of the study and variations in the seasonal occurrence of Ramadan (18). It is important to note in this connection that Ramadan is a lunar month, and every non lunar year it occurs 11 days earlier. So every 9 years, Ramadan occurs in a different season and duration of fasting and ambient temperature change continuously in the same region.

Several studies have been conducted in patients with diabetes especially type 2 diabetes. Some have reported an increase in the frequency of severe hypoglycaemia during Ramadan in these patients (25-27) and several others have confirmed the lack of effect of Ramadan fasting on blood glucose (28-30) and $\operatorname{HbA1C}(14,30,31)$. Others have found that $\mathrm{HbA1c}$ may even decrease during Ramadan $(22,28)$. A review of the literature (32) showed that the fasting month of Ramadan alter significantly glycemic control in type 2 diabetics if their glycaemia was not stable before the fasting; however, it seems to have little effects in patients with well equilibrated glycaemia before Ramadan. According to the results of our study, it seems that Ramadan fasting improves glycemic control. This effect seems most evident in diabetics.

The total energy intake in our study decreased during Ramadan and increased after Ramadan; all these changes were significant. These findings negate the common belief that Muslims tend to overcompensate in terms of food intake during Ramadan fasting month
(33). Overall changes of protein, fat and carbohydrate intake percentages were not significant and neither the glycaemia nor the lipid profile was correlated to the total energy intake. That proves that all the changes that we found early are due only to Ramadan fasting.

\section{Conclusion}

Our study findings support that beneficial effect of Ramadan fasting in elderly patients with cardiovascular risk factors were observed in early post-fasting period including an increase of HDL-C levels and better control of glycaemia. In contrast, in the fasting period there is a risk that renal function decrease with a simultaneous increase of glycaemia which could be deleterious for diabetic patients.

Funding: This study was supported by the Research Laboratory LR06SP21, Emergency Department, Pr. Semir Nouira, 5019, Monastir, Tunisia.

\section{Conflict of interest statement: There is no conflict of interest}

Ethical approval: The study received approval from the ethical Institutional Review Board of Fattouma Bourguiba University Hospital.

Acknowledgments: We thank all the members of Ramadan Research Group: Olfa Harzallah; Taher Chakroun; Nabil Sakly; Zohra Dridi; Sonia Hamdi; Khaldoun Ben Hamda; Asma Sriha; Samar Amor; Mounira Sahtout; Afifa Koubaa; Alia Haddad; Samia Grira; Néjia Bhouri; Ridha Boughraira; Habib Rafrafi; Olfa Bouraoui; Houda Haddad; Hichem Belhadj Youssef; Houda Ben Soltane; Mohamed Amine Msolli; Lamia Achour; Rached Bousrih; Hédi Majdoub; Mounira Attia; Fahima Hassine; Saousen Chouchène; Adel Ghali; Somaya Charfi; Fayrouz Elarem; Soukayna Achour.

\section{References}

1. Aslam M, Assad A. Drug Regimens and Fasting During Ramadan: A Survey in Kuwait. Public Health; 1986; 100: 49-53.

2. Iraki L, Bogdan A, Hakkou F, Amrani N, Abkari A, Touitou Y. Ramadan diet restrictions modify the circadian time structure in humans. A study on plasma gastrin, insulin, glucose, and calcium and on gastric $\mathrm{pH}$. J Clin Endocrinol Metab. 1997; 82: 1261-73.

3. Borgan A, Bouchareb B, Touitou Y. Ramadan fasting alters endocrine and neuroendocrine circadian patterns. Meal-time as a synchronizer in humans? Life Sci. 2001; 68: 1607-15

4. Bouzid C, Hssine M, Hajjem S, Saadallah S, Mhadhbi A, Turki Z, Ben Slama C. Paramètres anthropométriques et comportement alimentaire d'une population de sujets âgés tunisiens au cours du mois de Ramadan. Diabetes \& Metabolism, Volume 36, Supplement 1, 2010: A70.

5. Leiper JB, Molla AM, Molla AM. Effects on health of fluid restriction during fasting in Ramadan. European Journal of Clinical Nutrition. 2003; 57:30-8.

6. Sebbani M, El Ansari N, El Mghari G, Amine M. Food intake during the month of Ramadan in Moroccan patients with type 2 diabetes. East Mediterr Health J. 2013; 19:276-81.

7. Bener A, Hamad A, Fares A, Al-Sayed HM, Al-Suwaidi J. Is there any effect of Ramadan fasting on stroke incidence? Singapore Med J. 2006; 47:404-8.

8. Nematy M, Alinezhad-Namaghi M, Rashed MM, Mozhdehifard M, Sajjadi SS, Akhlaghi S, et al. Effects of Ramadan fasting on cardiovascular risk factors: a prospective observational study. Nutr J. 2012; 11:69.

9. Khafaji HA, Bener A, Osman M, Al Merri A, Al Suwaidi J. The impact of diurnal fasting during Ramadan on the lipid profile, hs-CRP, and serum leptin in stable cardiac patients. Vasc Health Risk Manag. 2012; 8:7-14.

10. Paltiel L, Rønningen KS, Meltzer HM, Baker SV, Hoppin JA. Evaluation of Freeze Thaw Cycles on stored plasma in the Biobank of the Norwegian Mother and Child Cohort Study. Cell Preserv Technol. 2008; 6:223-30.

11. El-Wakil HS, Desoky I, Lotfy N, Adam AG. Fasting the month of Ramadan by Muslims: could it be injurious to their kidneys? Saudi J Kidney Dis Transpl. 2007;18:349-54.

12. Maughan RJ, Leiper JB, Bartagi Z, Zrifi R, Zerguini Y, Dvorak J. Effect of Ramadan fasting on some biochemical and haematological parameters in Tunisian youth soccer players undertaking their usual training and competition schedule. J Sports Sci. 2008; Suppl 3:39-46. 
13. Trabelsi K, Stannard SR, Ghlissi Z, Maughan RJ, Kallel C, Jamoussi K, et al. Effect of fed versus fasted state resistance training during Ramadan on body composition and selected metabolic parameters in bodybuilders. J Int Soc Sports Nutr. 2013; 10:23.

14. Qurashi S, Tamimi A, Jaradat M, Al Sayyari A. Effect of fasting for Ramadan on kidney graft function during the hottest month of the year (August) in Riyadh, Saudi Arabia. Exp Clin Transplant. 2012; 10:551-3.

15. Kul S, Savaş E, Oztürk ZA, Karadağ G. Does Ramadan Fasting Alter Body Weight and Blood Lipids and Fasting Blood Glucose in a Healthy Population? A Meta-analysis. J Relig Health. 2014;53:929-42.

16. Temizhan A, Donderici O, Ouz D, Demirbas B. Is there any effect of Ramadan fasting on acute coronary heart disease events? Int J Cardiol.1999; 70:149-53.

17. Salim I, Al Suwaidi J, Ghadban W, Alkilani H, Salam AM. Impact of religious Ramadan fasting on cardiovascular disease: a systematic review of the literature. Curr Med Res Opin. 2013; 29: 343-54.

18. Sadiya A, Ahmed S, Siddieg HH, Babas IJ, Carlsson M. Effect of Ramadan fasting on metabolic markers, body composition, and dietary intake in Emiratis of Ajman (UAE) with metabolic syndrome. Diabetes Metab Syndr Obes. 2011; 4:409-16.

19. Bahijri S, Borai A, Ajabnoor G, Abdul Khaliq A, AlQassas I, Al-Shehri D, Chrousos G. Relative metabolic stability, but disrupted circadian cortisol secretion during the fasting month of Ramadan. PLoS One. 2013; 8:60917.

20. Barkia A, Mohamed K, Smaoui M, Zouari N, Hammami M, Nasri M. Change of diet, plasma lipids, lipoproteins, and fatty acids during Ramadan: a controversial association of the considered Ramadan model with atherosclerosis risk. J Health Popul Nutr. 2011; 29:486-93.

21. Lotfi S, Madani M, Tazi A, Boumahmaza M, Talbi M. Variation of cognitive functions and glycemia during physical exercise in Ramadan fasting. Rev Neurol (Paris). 2010; 166:721-6.

22. Norouzy A, Mohajeri SM, Shakeri S, Yari F, Sabery M, Philippou E, et al. Effect of Ramadan fasting on glycemic control in patients with Type 2 diabetes. J Endocrinol Invest. 2012; 35:766-71.

23. Ziaee V, Razaei M, Ahmadinejad Z, Shaikh H, Yousefi R, Yarmohammadi L, et al. The changes of metabolic profile and weight during Ramadan fasting. Singapore Med J. 2006; 47:409-14.
24. Shariatpanahi ZV, Shariatpanahi MV, Shahbazi S, Hossaini A, Abadi A. Effect of Ramadan fasting on some indices of insulin resistance and components of the metabolic syndrome in healthy male adults. Br J Nutr. 2008; 100:147-51.

25. Salti I, Bernard E, Detournay B, Bianchi-Biscay M. Le Brigand C, Voinet C et al. A population based study of diabetes and its characteristics during the fasting month of Ramadan in 13 countries: results of the epidemiology of diabetes and Ramadan 1422/2001 (EPIDAR) study. Diabetes Care. 2004; 27: 2306-11.

26. M'guil M, Ragala MA, El Guessabi L, Fellat S, Chraibi A, Chabraoui L, et al. Is Ramadan fasting safe in type 2 diabetic patients in view of the lack of significant effect of fasting on clinical and biochemical parameters, blood pressure, and glycemic control? Clin Exp Hypertens. 2008; 30:339-57.

27. Bravis V, Hui E, Salih S, Mehar S, Hassanein M, Devendra D. Ramadan Education and Awareness in Diabetes (READ) programme for Muslims with Type 2 diabetes who fast during Ramadan. Diabet Med. 2010; 27:327-31.

28. Aziz KM. Effect of Fasting Ramadan in Diabetes Control Status - Application of Extensive DiabetesEducation, Serum Creatinine with $\mathrm{HbA1c}$ Statistical ANOVA and Regression Models to Prevent Hypoglycemia. Recent Pat Endocr Metab Immune Drug Discov. 2013; 7:233-51.

29. Karatoprak C, Yolbas S, Cakirca M, Cinar A, Zorlu M, Kiskac M, et al. The effects of long term fasting in Ramadan on glucose regulation in type 2 Diabetes Mellitus. Eur Rev Med Pharmacol Sci. 2013; 17:2512-16.

30. Sahin SB, Ayaz T, Ozyurt N, Ilkkilic K, Kirvar A, Sezgin H. The Impact of Fasting during Ramadan on the Glycemic Control of Patients with Type 2 Diabetes Mellitus. Exp Clin Endocrinol Diabetes. 2013;121:531-4.

31. Bouguerra R, Jabrane J, Maâtki C, Ben Salem L, Hamzaoui J, El Kadhi A et al. La pratique du jeûne du mois de Ramadan chez le diabétique de type 2 . Annales d'Endocrinologie. 2006; 67: 54-9.

32. Benaji B, Mounib N, Roky R, Aadil N, Houti IE, Moussamih S et al. Diabetes and Ramadan: Review of the literature. Diabetes Research and Clinical Practice. 2006; 73: 117-25.

33. Wissam HI, Hosam MH, Amjad HJ, Samer AAB. Effect of Ramadan fasting on markers of oxidative stress and serum biochemical marker of cellular damage in healthy subjects. Ann Nutr Metab. 2008; 53: 175-81. 